\title{
EXPLORING THE TRADE-SPACE OF MIMO SAR
}

\author{
Marwan Younis, Paco López-Dekker, Federica Bordoni, Piotr Laskowski, and Gerhard Krieger
}

German Aerospace Center (DLR), Microwaves and Radar Institute, Germany

\section{ABSTRACT}

The term MIMO SAR is used for Synthetic Aperture Radar (SAR) systems utilizing multiple-receive and multiple-transmit channels (Multiple-Input Multiple Output: MIMO). The trade-space for such SAR systems includes the instrument and antenna parameters taking into consideration the techniques, i.e. the operation modes of the radar. The purpose of exploring this trade-space is to improve and optimize the performance of the system, described through the performance parameters.

The aim of this paper is to show examples of how MIMO SAR offers new and unexpected ways of trading the system and performance parameters versus each other. Adequately exploring the trade-space yields a high flexibility and allows to simultaneously improve multiple performance parameters; a feature not available in conventional single channel SAR systems. The content of this paper is considered novel in the sense that it deals with highly advance SAR techniques.

\section{INTRODUCTION}

The main innovative characteristic of forthcoming generations of SAR systems is the use of multiple elevation and/or azimuth receiver channels combined with digital beamforming (DBF) capability [1, 2, 3]. This allows for the synthesis of multiple dynamic digital receiver beams. Further, multiple transmit channels are being suggested as an extension to DBF systems. Adding transmitters can be beneficial as it offers interferometric capabilities or allows using sub-pulse techniques [4]. With this, we arrive at what is commonly referred to as MIMO SAR (Multiple-Input Multiple-Output SAR), a term originating from communication.

The virtue of MIMO SAR is that it extends the dimension of the trade-space. This allows the conception of systems which overcome or bypass the limitation of conventional SAR. Obviously multi-channel SAR offer the engineers a wide variety of options for constructing new operation techniques (modes) and combining them in multiple ways. The question is whether these techniques are useful in the sense of improving the performance? Indeed there are several published MIMO operation techniques which turn out to increase the complexity while in the end not improving the performance.
The paper is organized as follows: in the next section a set of parameters is introduced which describes the SAR system and its performance. In section 4 a MIMO SAR system is introduced which serves as an example for demonstrating various approaches in section 5 which explore the trade-space. Interesting and unexpected compromises will be given for realistic SAR missions. The approach is to keep the description on a system level, thus avoiding too much detail.

\section{SAR TRADE-SPACE PARAMETERS}

The most relevant system and performance parameters are shown in Fig. 1. Here the system parameters basically describe the instrument both in terms of quantities fixed to the system design such as antenna dimensions, as well as operation parameters which can be set for a specific data take (e.g. the Pulse Repetition Frequency, PRF). The performance is usually derived from the mission requirement and described in terms of a set of values for the performance parameters [5].

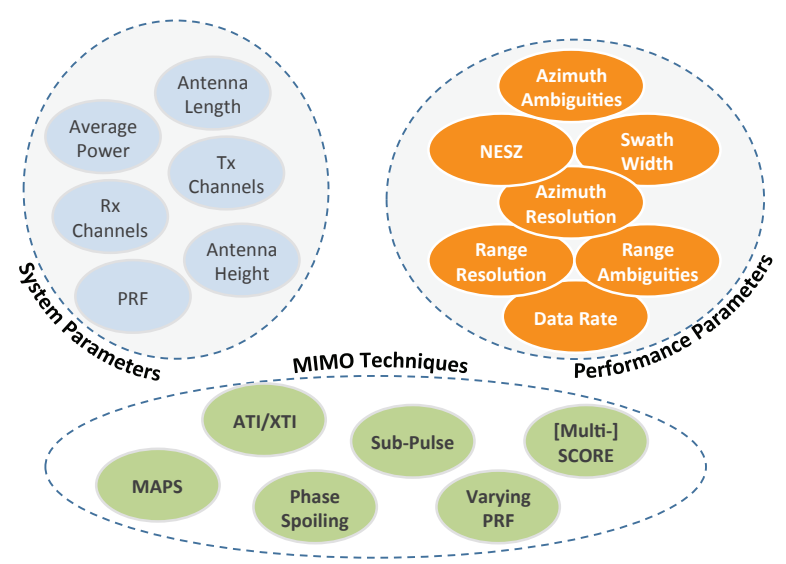

Fig. 1. The most relevant system and performance parameters for SAR. These parameters can be optimized using different techniques (modes).

\section{EXAMPLE MIMO SYSTEM}

The specific system configuration to demonstrate each trade tends to be different depending on the parameters involved. 
Here we present one generic system which serves as an example to demonstrate most of the trades. This system is shown in Fig. 2 and consists of two transmit reflector antennas with fixed beams and a single receive reflector with a digital multichannel feed array. A detailed description of the system and its performance (although not necessary for the understand the subsequent sections) is given in $[6,7]$

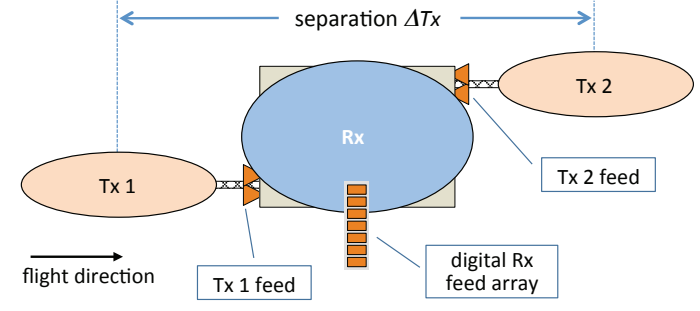

Fig. 2. Example MIMO SAR system showing the configuration consisting of two fixed beam Tx reflector antennas and a single Rx reflector utilizing a digital feed.

\section{MIMO SAR TRADE-SPACE EXAMPLES}

In the following a few interesting examples are given of how the trade-space parameters interact.

\subsection{Classical Trade: Resultion versus Swath}

To understand the trade-space parameters we consider the classical trade between swath and resolution. The process for improving the azimuth resolution for a conventional singlechannel SAR is shown in Fig. 3(a) where the interaction between system and performance parameters finally leads to a reduced swath width. Similarly, any attempt to increase the swath width leads to a worsening of the azimuth resolution. In both cases the reason can be traced back to Shannon's sampling theorem, which gives the minimum number of azimuth samples per time interval.
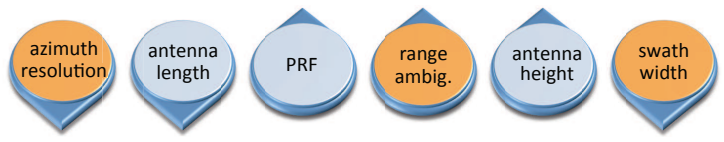

(a) conventional single channel SAR
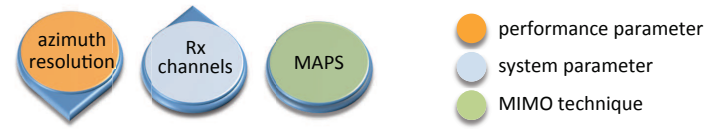

(b) multi-azimuth receive channel SAR

Fig. 3. The interaction between the various trade-space parameters as a result of improving the azimuth resolution. Here the parameter type is color coded, while up/down arrows indicate whether the respective value increases or decreases.
The High-azimuth-Resolution and Wide-Swath SAR also known as HRWS [8, 9] overcomes this limitation. By dividing the receive antenna into multiple sub-apertures and recording the data from each of them the effective sampling rate is increased. This improves the azimuth resolution without sacrificing swath width ${ }^{1}[10,2]$ but requires a dedicated processing $[11,3]$. Note that a higher, i.e. better, resolution implies a smaller value of the resolution parameter.

\subsection{Antenna Height versus Swath Width}

Going back to a single channel SAR it is noticed that the antenna height is inversely proportional to the swath width. This is because the swath is determined by the beam footprint on the ground which is given by the ratio of wavelength to antenna height $\lambda / h_{0}$. Increasing the swath requires a reduced antenna height which has consequences on the azimuth resolution as shown before.

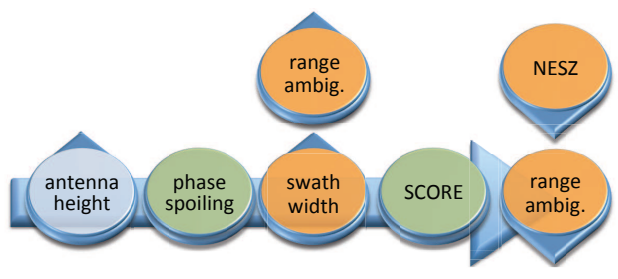

Fig. 4. Trade-space example where increasing the antenna height leads to an increased swath width.

This dependency is reversed for a MIMO SAR, i.e. an increased swath requires a higher antenna as supported by the following arguments. The system designer will choose to increase the antenna height such that $h>h_{0}$; to ensure illuminating a larger footprint phase spoiling will be used in case of a planar antenna, while in the case of a reflector the elevation beamwidth is increased by narrowing the primary feed's beamwidth. The appearing range ambiguities will be suppressed by using digital beamforming on-receive in elevation. The basic technique is known as SCan-On-REceive (SCORE) [12] where a narrow receive beam follows the pulse echo on the ground in real time (see Fig. 6 and discussion in section 5.3). Taking a closer look at the power levels will show multiple effects: The power density on the ground inevitable drops due to the larger Tx beamwidth and the associated reduced antenna gain; however the increased number of transmit-receive modules involved causes an increase in the total Tx power; on receive the system profits from the increased gain due to the high antenna, which improves the Rx power. The trade-space parameter interaction in shown in Fig. 4

\footnotetext{
${ }^{1}$ assuming that the complexity, i.e. number of $\mathrm{Rx}$ channels is not a performance parameter.
} 


\subsection{Azimuth Resolution versus Average Power}

In MIMO SAR the average power can be increased to improve the azimuth resolution. To show this, consider a single channel reflector based SAR with separate Tx and Rx antennas designed to yield an azimuth resolution $\delta_{a z 0}$. Now, a second Tx antenna is added [7, 6] as shown in Fig. 2. The system is operated such that two sub-pulses are transmitted within each pulse repetition interval (PRI). This operation technique is known as the sub-pulse mode [4] where the two sub-pulses are usually delayed by a small fraction of the PRI with respect to each other as shown in Fig. 5. As a result of doubling the number of transmitted pulses within the same time interval the total average Tx power is doubled when compared to the initial system. But now the number of received samples per PRI is also doubled (i.e. the spacial sampling is doubled) which reflects the unambiguous Doppler bandwidth and by this the azimuth resolution ${ }^{2}$ which becomes $\delta_{a z}=\delta_{a z 0} / 2$.

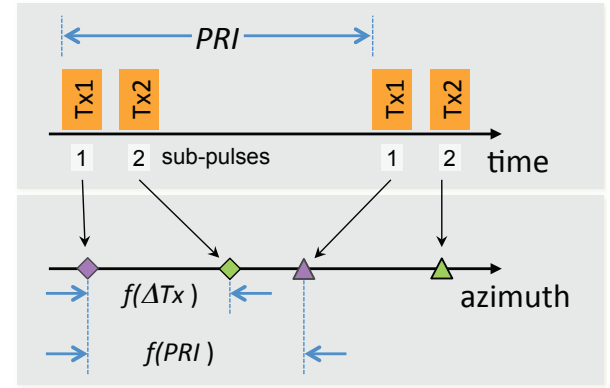

Fig. 5. The position of the sub-pulse samples depends on the Tx antenna separation and the PRI.

The echo signals of the two sub-pulses arrive at nearly the same time at the receiver. To separate the two echos multiSCORE is utilized here (see Fig. 6), where two receive beams are generated, each one maximized to the direction of arrival of one sub-pulse while suppressing the energy of the other [4].

Note that the SAR system described here is truly a MIMO SAR utilizing both multiple-transmit and multiple-receive channels.

\subsection{Interferometry versus Power}

By increasing the PRF for the previous MIMO SAR the spatial samples of subsequent pulses coincides (c.f. Fig. 7) thus enabling along-track interferometric (ATI) capabilities. Similarly if the arrangements of the Tx antennas is changed to be displaced vertically instead of horizontally, cross-track interferometry (XTI) is provided. In both cases when comparing to a system with one Tx and two Rx antennas, the actual trade is power versus interferometric capabilities.

\footnotetext{
${ }^{2}$ Of course this requires that the antenna lengths are adapted to match the increased Doppler bandwidth.
}

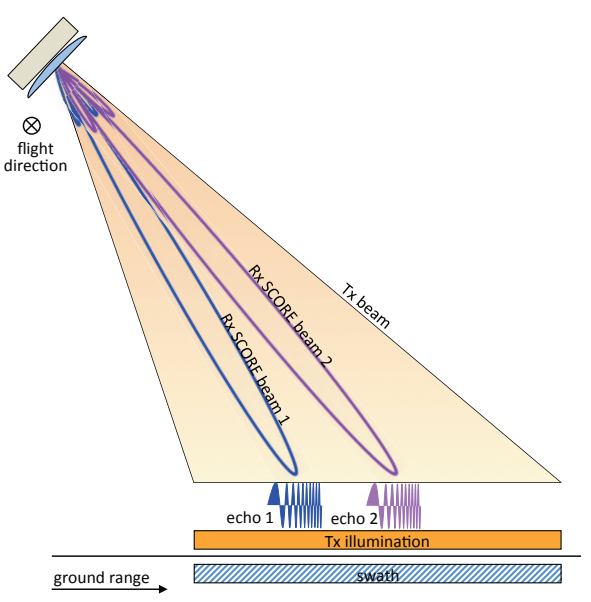

Fig. 6. Multi-SCORE operation mode where two sub-pulses are tracked simultaneously.

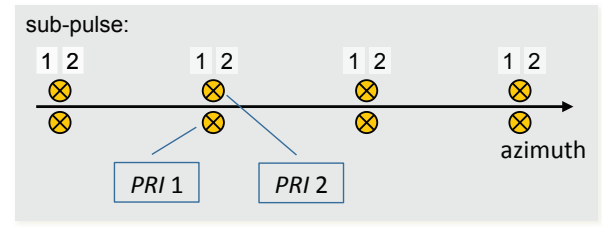

Fig. 7. By adjusting the pulse repetition frequency the first sub-pulse of a PRI can postioned such that it coincides with the second sub-pulse of the previous PRI.

\subsection{Range Resolution versus Swath Width}

Another possibility for increasing the swath width of a MIMO SAR is to split the available bandwidth $B_{0}$ into multiple $M$ sub-bands such that each sub-band is dedicated to one subswath. The digital beamforming capabilities on transmit are used to transmit chirp waveforms each of bandwidth $B=$ $B_{0} / M$ to different angular segments. The bandwidths of the different chirps do not overlap, i.e. the center frequency of each chirp differs slightly from that of the others. The receive bandwidth is $B_{0}$ thus simultaneously collecting and sampling the echos from the sub-swathes which may arrive at the same time. The waveforms are easily separated before processing using digital bandpass filters.

\subsection{Data Rate versus Swath Width}

In general when increasing the imaged swath width of a SAR system two aspects have to be considered: the range ambiguities and the timing. The former can be controlled by using one of the techniques described before. The timing restriction is caused by the fact that pulsed SAR systems are generally not designed to transmit and receive at the same time. The echo window time, determined by the $P R F_{0}$, the pulse duty cycle, and the orbital geometry give the time where the echo 
signal can actually be received. Thus the pulse transmit times cause gaps in the data samples of the received echo and consequently "blind ranges" in the resulting image.

One possibility to overcome blind ranges is to vary the PRI from pulses-to-pulse [13] which smears the blind ranges across the swath. However, in order to maintain the quality of the azimuth impulse response, it turns out that the average PRF must be increased, i.e. $\overline{P R F}>P R F_{0}$. The ratio of average PRF to processed Doppler bandwidth is known as the oversampling factor $\gamma=\overline{P R F} / B_{\text {Doppler }}$ which will be increased and is $>1$. The data rate on the other hand is obviously proportional to the swath width being imaged, however the value of $\gamma$ enters the equation for the data rate as an additional multiplicative factor. In a way it could be understood as the ration of data rate to information rate.

\section{CONCLUSION}

The trade parameters of an example MIMO SAR system using two transmitters separated in along-track direction and one receive with multiple elevation channels was examined. It was shown that MIMO SAR offers a high flexibility in trading the various parameters. Examples were given where changing the value of a system parameter produced the opposite results than what is known from classical single channel SAR.

\section{REFERENCES}

[1] G. Krieger, N. Gebert, and A. Moreira, "Multidimensional waveform encoding: A new digital beamforming technique for synthetic aperture radar remote sensing," IEEE Transactions on Geoscience and Remote Sensing, vol. 46, no. 1, pp. 31-46, Jan. 2008.

[2] M. Younis, "Digital beam-forming for high resolution wide swath real and synthetic aperture radar," $\mathrm{Ph}$.D. dissertation, Institut für Höchstfrequenztechnik und Elektronik, Universität Karlsruhe, Jul. 2004.

[3] N. Gebert, G. Krieger, and A. Moreira, "Digital beamforming on receive: Techniques and optimization strategies for high-resolution wide-swath SAR imaging," IEEE Transactions on Aerospace and Electronic Systems, vol. 45, pp. 564-592, 2009.

[4] F. Bordoni, M. Younis, and G. Krieger, "Performance investigation on the high-resolution wide-swath SAR system operating in multisubpulse mode," in Proc. Int. Geoscience and Remote Sensing Symposium IGARSS'2012, Munich, Germany, Jul. 2012.

[5] M. Younis, S. Huber, A. Patyuchenko, F. Bordoni, and G. Krieger, "Performance comparison of reflector- and planar-antenna based digital beamforming SAR," Int. Journal of Antennas and Propagation, vol. 2009, Jun. 2009. [Online]. Available: http://www.hindawi.com/journals/ijap/2009

[6] M. Younis, P. López-Dekker, A. Patyuchenko, and G. Krieger, "Digital beamforming architecture and techniques for a spaceborne interferometric ka-band mission," in Proceedings of the IEEE Radar Conference, Ottawa, Canada, Apr. 2013.

[7] P. López-Dekker, M. Younis, J. A. García, T. Börner, and G. Krieger, "Advanced digital beamforming architectures and operation modes for an enhanced SIGNAL mission concepts," in Proc. 1st workshop on Ka-band Earth Observation Radar Missions (KEO'12), Noordwijk, The Netherlands, Nov. 2012.

[8] C. Fischer, C. Schaefer, and C. Heer, "Technology development for the HRWS (High Resolution Wide Swath) SAR," in International Radar Symposium IRS'07, Sep. 2007.

[9] M. Süß, M. Zubler, and R. Zahn, "Performance investigation on the high resolution, wide swath SAR system," in Proc. European Conference on Synthetic Aperture Radar EUSAR'2002, Koeln, Germany, Jun. 2002, pp. 187-190.

[10] G. D. Callaghan, "Wide-swath space-borne SAR: Overcoming the trade-off between swath-width and resolution," Ph.D. dissertation, University of Queensland's, 1999.

[11] G. Krieger, N. Gebert, and A. Moreira, "Unambiguous SAR signal reconstruction from non-uniform displaced phase centre sampling," IEEE Geoscience and Remote Sensing Letters, vol. 1, no. 4, pp. 260-264, Oct. 2004.

[12] M. Suess and W. Wiesbeck, "Side-looking synthetic aperture radar system," European Patent Patent EP 1241 487, Sep., 2002.

[13] M. Villano, G. Krieger, and A. Moreira, "Staggered SAR: High-resolution wide-swath imaging by continuous PRI variation," submitted to IEEE Transactions on Geoscience and Remote Sensing, 2013. 